\title{
Flavonoids, Phenylethanoids and Phenylpropanoids from Rhodiola rosea L. and Their Lipid-Lowering Activities on Receptor 5- $\mathrm{HT}_{2 \mathrm{~A}}$
}

\author{
Hao Wang, ${ }^{a, b}$ Ji-Lei Liang, ${ }^{a}$ Qing-Ming He, ${ }^{a}$ Guo-Hai Zhou, ${ }^{a}$ Cun-Fu Li, ${ }^{a}$ Ping-He Wei, ${ }^{a}$ and Hao-Yan Dong ${ }^{\star, a}$ \\ a Jiangsu Key Laboratory of Chiral Pharmaceuticals Biosynthesis, Taizhou University, Taizhou, Jiangsu 225300, \\ China \\ ${ }^{b}$ DICP-CMC Innovation Institute of Medicine, Taizhou, Jiangsu 225300, China \\ Email: donghaoyan2008@126.com (H. Y. D.)
}

\begin{abstract}
Fifteen compounds, including 11 flavonoids, 2 phenylethanoids and 2 phenylpropanoids, were isolated from the roots of Rhodiola rosea L. Among them, quercetin (1) and quercetin-3-O- $\beta$ - $D$-glucoside (2) were afforded from the genus Rhodiola for the first time. All isolates were evaluated for their agitating activities on receptor $5-\mathrm{HT}_{2 \mathrm{~A}}$ and four compounds $(\mathbf{1 2}-\mathbf{1 5})$ showed obvious agitating activities, which were probably the main lipid-lowering active components of $R$. rosea $\mathrm{L}$.
\end{abstract}

Keywords flavonoids, phenylpropanoids, phenylethanoids, Rhodiola rosea, lipid-lowering activity, receptor $5-\mathrm{HT}_{2 \mathrm{~A}}$

\section{Introduction}

Obesity, mainly caused by over nutrition and inheritance, can give rise to hypertension, hyperlipidemia and heart diseases, which threatens human health directly or potentially. ${ }^{[1]}$ Lipid regulation is one of the most effective measures that have been developed to deal with obesity. ${ }^{[1,2]}$

Rhodiola rosea L., a medicine-food resource plant that grows primarily in mountainous and arctic regions, is widely used as folk medical systems throughout the eastern Europe and Asia to improve resistance to high altitude sickness, stimulate the nervous system and treat fatigue, psychological stress and depression. ${ }^{[3]}$ The main active mechanism of its anti-allergenic, anti-inflammatory, anti-depressant, anti-oxidant and lipid-lowering activities revealed by pharmacological studies is improving human immunity and strengthening the human self-regulation systems. ${ }^{[3-5]}$ Besides, compounds reported from $R$. rosea L. contain flavonoids, monoterpenes, triterpenes, phenolic acids along with the specific components of this plant, phenylethanoids (tyrosol and salidroside) and phenylpropanoids (rosin) ${ }^{[3,4]}$

As a member of serotonin (5-HT) receptors, receptor $5-\mathrm{HT}_{2 \mathrm{~A}}$ has been found to mediate a variety of serotonin's physiological functions in both the central and peripheral nervous systems, and its selective agonist is a useful therapeutic approach for certain disorders, including hypertension, hyperlipidemia and obesity. ${ }^{[5]}$ Additionally, it was reported that high activity of $5-\mathrm{HT}_{2 \mathrm{~A}}$ receptor can reduce appetite and food intake to achieve the purpose of lipid regulation. ${ }^{[2,5,6]}$

To clarify the lipid-lowering components of the entitled plant, the bioassay-guided investigation was carried out, which led to isolation of 11 flavonoids (Figure 1), 2 phenylethanoids and 2 phenylpropanoids. Among them, flavones 1-2 were isolated from the Rhodiola genus for the first time.

\section{Experimental}

\section{General apparatus and chemicals}

${ }^{1} \mathrm{H}$ NMR and ${ }^{13} \mathrm{C}$ NMR experiments were performed using

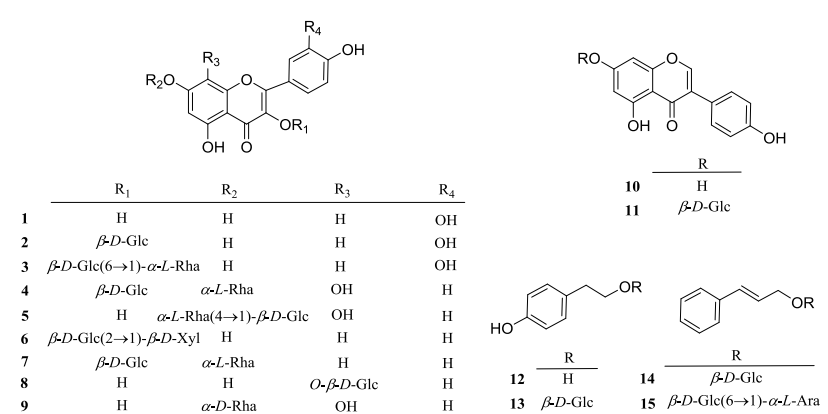

Figure 1 Structures of flavonoids, phenylethanoids andphenylpropanoids isolated from $R$. rosea $\mathrm{L}$.

Avance III-600 NMR spectrometers (Bruker, Bremerhaven, Germany) with tetramethylsilane as the internal standard. Preparative HPLC was carried out on Newstyle ${ }^{\mathrm{TM}}$ equipment (NP-7000 pump, NU-3000 detector; Hanbon Sci. \& Tech., China) with a dynamic axial compression column $(50 \mathrm{~mm}$, Hanbon Sci. \& Tech., China) filled with $C_{18}(10 \mu \mathrm{m}$, Agilent USA). Semi-preparative HPLC was performed on a Newstyle ${ }^{T M}$ (pump: NP-7000 serials, detector: NU-3000 serials) with a reversed-phase $(\mathrm{RP}) \mathrm{C}_{18}$ column $(9.4 \times 250 \mathrm{~mm}, 5 \mu \mathrm{m})$. HPLC analysis was recorded on Waters E 2695 Separations Module (2998 PDA detector, Waters, USA) with a $\mathrm{C}_{18}$ column $(4.5 \times 250$ $\mathrm{mm}, 5 \mu \mathrm{m}$, Agilent, USA). Absorption values were read with Flex Station 3 Benchtop Multi-Mode Microplate Reader (Molecular Devices, Sunnyvale, California, USA). Serotonin hydrochloride (purity > 99\%) was purchased from Alfa Aesar as positive control.

\section{Plant material}

The roots of $R$. rosea $\mathrm{L}$. was purchased from the Yuzhou traditional Chinese medicine market (Yuzhou, Henan, China) in January 2018 and identified by Professor Jia-Mei Li in Henan Agricultural University. A voucher specimen (No. 2018-08-21) was deposited in the Jiangsu Key Laboratory of Chiral Pharmaceuticals Biosynthesis, Taizhou University. 


\section{Extraction and isolation}

The air-dried and powdered root of $R$. rosea $\mathrm{L}$. (1 kg) was extracted with $70 \% \mathrm{EtOH}$ at room temperature. The extracts were combined, condensed in vacuo, filtrated by $500 \mathrm{~nm}$ ceramic membrane, and then the filtrate was loaded and partitioned by preparative HPLC to give water soluble fraction, $50 \% \mathrm{EtOH}$ fraction and $95 \% \mathrm{EtOH}$ fraction. The 50\% EtOH fraction $(82 \mathrm{~g})$ with an agonistic rate of $38.1 \%$ on the receptor $5-\mathrm{HT}_{2 \mathrm{~A}}$ at the concentration of $1.02 \mathrm{mg} / \mathrm{mL}$ was chosen for further isolation following bioassay tests (Table $\mathrm{S} 1$ in Supporting Information).

The $50 \% \mathrm{EtOH}$ fraction $(82 \mathrm{~g})$ was subjected to preparative HPLC and eluted with $\mathrm{MeOH}-\mathrm{H}_{2} \mathrm{O}-\mathrm{HAc}(20: 80: 0.08,40: 60:$ $0.06,60: 40: 0.04,80: 20: 0.02, V / V / V$, each $4 \mathrm{CV}$ ) mixtures to afford the fractions Frs.1-4. Fr.1 was further fractionated and purified by repeated preparative HPLC and semi-preparative HPLC to obtain the target compound 4 (13 $\mathrm{mg}$ ). Fr.2 was divided into four fractions Frs.2-1-2-4 by gradient elution using $\mathrm{MeCN}-\mathrm{H}_{2} \mathrm{O}(20: 80-35: 65)$, then Fr.2-2 was further purified by repeated semi-preparative HPLC for compound 9 (6 mg), and Fr.2-3 was further partitioned to yield compounds $6(12 \mathrm{mg})$ and $7(21 \mathrm{mg})$. Fr.3 was successively partitioned into eight sub-fractions Frs.3-1-3-8 eluted by $\mathrm{MeCN}-\mathrm{H}_{2} \mathrm{O}$ (45: 55-55:45), and every sub-fraction was further purified by repeated semi-preparative HPLC for compounds 2 (12 mg), 3 (31 mg), 5 (49 mg), 8 (7 mg), 11 (8 $\mathrm{mg}), 13(217 \mathrm{mg}), 14(86 \mathrm{mg})$ and $15(69 \mathrm{mg})$. Fr. 4 was purified repeatedly by semi-preparative HPLC to obtain compounds 1 (9 $\mathrm{mg}), 10(16 \mathrm{mg})$ and $12(142 \mathrm{mg})$. The purities of all titled compounds were $>98 \%$ determined by HPLC.

\section{Agonistic activity assay}

$\mathrm{HEK} 293$ cell line was cultured with $5 \% \mathrm{CO}_{2}$ at $37{ }^{\circ} \mathrm{C}$ in DMEM supplemented with $10 \%$ FBS. The cells were seeded in a Matrigel coated 96-well black wall/clear bottom plate following been incubated in $\mathrm{CO}_{2}$ incubator for $24 \mathrm{~h}$, dyed by HDB Wash Free Fluo- 8 Calcium Assay kit at $37^{\circ} \mathrm{C}$ in dark for one hour, and then treated with tested compounds and positive drug, respectively. The absorption values were read with Flex Station 3 Benchtop Multi-Mode Microplate Reader at room temperature with excitation wavelength at $485 \mathrm{~nm}$, emission wavelength at $525 \mathrm{~nm}$ and emission cut-off at $515 \mathrm{~nm}$. The agonistic rates of the test compounds were calculated by comparing with the positive control serotonin hydrochloride, whose agonistic rate was normalized to $100 \%{ }^{[7]}$

\section{Results and Discussion}

Investigation on the roots of $R$. rosea $\mathrm{L}$. afforded 15 compounds including 11 flavonoids, 2 phenylethanoids and 2 phenylpropanoids. Among them, flavones 1-2 were isolated from the Rhodiola genus for the first time. As shown in Figure 1, the structures of these compounds were elucidated by comparing their ${ }^{1} \mathrm{H}$ NMR and ${ }^{13} \mathrm{C}$ NMR spectra with the reported literatures.

Quercetin (1): ${ }^{[8]}$ yellowish powder, $\mathrm{C}_{15} \mathrm{H}_{10} \mathrm{O}_{7},{ }^{1} \mathrm{H}$ NMR $(600$ $\left.\mathrm{MH}_{\mathrm{z}}, \mathrm{CD}_{3} \mathrm{OD}\right) \delta: 7.67(1 \mathrm{H}, \mathrm{d}, J=2.0 \mathrm{~Hz}), 7.58(1 \mathrm{H}, \mathrm{dd}, J=8.5$, $2.0 \mathrm{~Hz}), 6.88(1 \mathrm{H}, \mathrm{d}, J=8.5 \mathrm{~Hz}), 6.41(1 \mathrm{H}, \mathrm{d}, J=1.8 \mathrm{~Hz}), 6.19$ $(1 \mathrm{H}, \mathrm{d}, J=1.8 \mathrm{~Hz}) ;{ }^{13} \mathrm{C}$ NMR $\left(150 \mathrm{MH}_{\mathrm{z}}, \mathrm{CD}_{3} \mathrm{OD}\right) \delta: 175.7$ (s), 163.8 (s), 160.6 (s), 156.1 (s), 147.6 (s), 146.6 (s), 145.0 (s), 135.6 (s), 122.0 (s), 119.8 (d), 115.5(d), 115.0 (d), 102.8 (s), $98.0(d), 93.2(d)$.

Quercetin-3-O- $\beta$ - $D$-glucoside (2): $:^{[9]}$ yellow powder, $\mathrm{C}_{21} \mathrm{H}_{20} \mathrm{O}_{12},{ }^{1} \mathrm{H}$ NMR $\left(600 \mathrm{MHz}, \mathrm{CD}_{3} \mathrm{OD}\right) \delta: 7.71(1 \mathrm{H}, \mathrm{d}, J=2.0$ $\mathrm{Hz}), 7.58(1 \mathrm{H}, \mathrm{dd}, J=8.4,2.0 \mathrm{~Hz}), 6.86(1 \mathrm{H}, \mathrm{d}, J=8.4 \mathrm{~Hz}), 6.35$ $(1 \mathrm{H}, \mathrm{d}, J=1.9 \mathrm{~Hz}), 6.18(1 \mathrm{H}, \mathrm{d}, J=1.9 \mathrm{~Hz}), 5.23(1 \mathrm{H}, \mathrm{d}, J=7.6$ $\mathrm{Hz}), 3.71(1 \mathrm{H}, \mathrm{dd}, J=11.9,2.1 \mathrm{~Hz}), 3.57(1 \mathrm{H}, \mathrm{dd}, J=11.9,5.3$
$\mathrm{Hz}), 3.42-3.48(2 \mathrm{H}, \mathrm{m}), 3.34-3.37(1 \mathrm{H}, \mathrm{m}), 3.18-3.23(1 \mathrm{H}$, $\mathrm{m}) ;{ }^{13} \mathrm{C}$ NMR (150 MHz, $\left.\mathrm{CD}_{3} \mathrm{OD}\right) \delta: 179.2$ (s), 166.1 (s), 162.9 (s), 158.9 (s), $158.2(\mathrm{~s}), 149.7$ (s), 145.8 (s), 135.4 (s), 123.3 (d), 123.1 (s), 117.6 (d), 116.0 (d), 105.5 (s), 104.1 (s), 99.9 (d), $94.5(\mathrm{~d}), 78.1$ (d), 78.0 (d), $75.6(\mathrm{~d}), 71.1(\mathrm{~d}), 62.5(\mathrm{t})$.

Rutin (3): ${ }^{[10]}$ yellowish powder, $\mathrm{C}_{27} \mathrm{H}_{30} \mathrm{O}_{16},{ }^{1} \mathrm{H}$ NMR $(600$ $\left.\mathrm{MHz}, \mathrm{CD}_{3} \mathrm{OD}\right) \delta: 7.88(1 \mathrm{H}, \mathrm{d}, J=1.4 \mathrm{~Hz}), 7.58(1 \mathrm{H}, \mathrm{dd}, J=8.5$, $1.4 \mathrm{~Hz}), 6.85(1 \mathrm{H}, \mathrm{d}, J=8.5 \mathrm{~Hz}), 6.43(1 \mathrm{H}, \mathrm{d}, J=1.2 \mathrm{~Hz}), 6.21$ $(1 \mathrm{H}, \mathrm{d}, J=1.2 \mathrm{~Hz}), 5.05(1 \mathrm{H}, \mathrm{d}, J=7.8 \mathrm{~Hz}), 4.51(1 \mathrm{H}, \mathrm{s})$, $3.31-4.63(10 \mathrm{H}, \mathrm{m}), 1.15(3 \mathrm{H}, \mathrm{d}, J=6.6 \mathrm{~Hz}) ;{ }^{13} \mathrm{C}$ NMR $(150$ $\mathrm{MHz}, \mathrm{CD}_{3} \mathrm{OD}$ ) $\delta: 179.6$ (s), 166.1 (s), 163.1 (s), 158.7 (s), 158.4 (s), 150.1 (s), 145.6 (s), 135.6 (s), 122.9 (d), 122.7 (s), 117.8 (d), 116.0 (d), 105.9 (s), 105.3 (s), 101.8 (s), 99.9 (d), 94.5 (d), 75.2 (d), 75.0 (d), 73.8 (d), 73.0 (d), 72.2 (d), 71.8 (d), 70.1 (d), 69.5 (d), $67.2(\mathrm{t}), 18.1$ (q).

Herbacetin-3-O- $\beta$ - $D$-glucopyranosyl-7-O- $\alpha$ - $L$-rhamnopyranoside (4): ${ }^{[11]}$ white powder, $\mathrm{C}_{27} \mathrm{H}_{30} \mathrm{O}_{16},{ }^{1} \mathrm{H}$ NMR $(600 \mathrm{MHz}$, $\left.\mathrm{CD}_{3} \mathrm{OD}\right) \delta$ : $8.09(2 \mathrm{H}, \mathrm{d}, J=8.5 \mathrm{~Hz}), 7.02(2 \mathrm{H}, \mathrm{d}, J=8.5 \mathrm{~Hz})$, $6.71(1 \mathrm{H}, \mathrm{s}), 5.51(1 \mathrm{H}, \mathrm{d}, J=1.4 \mathrm{~Hz}), 5.28(1 \mathrm{H}, \mathrm{d}, J=7.5 \mathrm{~Hz})$, 3.36-4.51 $(10 \mathrm{H}, \mathrm{m}), 1.25(3 \mathrm{H}, \mathrm{d}, J=6.5 \mathrm{~Hz}) ;{ }^{13} \mathrm{C}$ NMR $(150$ $\mathrm{MHz}, \mathrm{CD}_{3} \mathrm{OD}$ ) $\delta: 177.1$ (s), 160.9 (s), 157.0 (s), 152.3 (s), 151.0 (s), 144.4 (s), 133.4 (s), 131.4 (d), 126.9 (s), 121.2 (s), 114.9 (d), 105.2 (s), 101.0 (d), 99.4 (d), 99.2 (d), 77.5 (d), 76.3 (d), 74.4 (d), 72.3 (d), 70.6 (t), 70.4 (d), 70.2 (d), 70.1 (d), 61.0 (t), 18.1 (q).

Rhodiosin (5): $:^{[3]}$ yellowish powder, $\mathrm{C}_{27} \mathrm{H}_{30} \mathrm{O}_{16},{ }^{1} \mathrm{H}$ NMR $(600$ $\left.\mathrm{MHz}, \mathrm{CD}_{3} \mathrm{OD}\right) \delta: 8.15(2 \mathrm{H}, \mathrm{d}, J=8.6 \mathrm{~Hz}), 6.88(2 \mathrm{H}, \mathrm{d}, J=8.6$ $\mathrm{Hz}), 6.62(1 \mathrm{H}, \mathrm{s}), 5.54(1 \mathrm{H}, \mathrm{d}, J=1.9 \mathrm{~Hz}), 4.67(1 \mathrm{H}, \mathrm{d}, J=7.1$ $\mathrm{Hz}), 4.18-4.30(2 \mathrm{H}, \mathrm{m}), 3.67-3.81(4 \mathrm{H}, \mathrm{m}), 3.35-3.42(4 \mathrm{H}$, $\mathrm{m}), 1.28(3 \mathrm{H}, \mathrm{d}, J=6.2 \mathrm{~Hz}) ;{ }^{13} \mathrm{C}$ NMR $\left(150 \mathrm{MHz}, \mathrm{CD}_{3} \mathrm{OD}\right) \delta$ : 176.8 (s), 160.3 (s), 153.2 (s), 150.8 (s), 148.6 (s), 145.7 (s), 137.1 (s), 130.8 (d), 128.4 (s), 123.6 (s), 116.2 (d), 106.1 (d), 105.5 (s), 100.3 (d), 99.0 (d), 82.1 (d), 77.6 (d), 77.5 (d), 75.3 (d), 72.2 (d), 71.1 (d), 71.0 (d), 70.6 (d), 62.3 (t), 18.2(q).

Kaempferol-3- $O-\beta$ - $D$-glucopyranoside- $(2 \rightarrow 1)$ - $D$-xylopyrano side (6): $:^{[12]}$ yellowish powder, $\mathrm{C}_{26} \mathrm{H}_{28} \mathrm{O}_{15},{ }^{1} \mathrm{H}$ NMR $(600 \mathrm{MHz}$, $\left.\mathrm{CD}_{3} \mathrm{OD}\right) \delta: 8.05(2 \mathrm{H}, \mathrm{d}, J=8.5 \mathrm{~Hz}), 6.88(2 \mathrm{H}, \mathrm{d}, J=8.5 \mathrm{~Hz})$, $6.39(1 \mathrm{H}, \mathrm{d}, J=1.9 \mathrm{~Hz}), 6.20(1 \mathrm{H}, \mathrm{d}, J=1.9 \mathrm{~Hz}), 5.50(1 \mathrm{H}, \mathrm{d}, J=$ $5.9 \mathrm{~Hz}), 4.72(1 \mathrm{H}, \mathrm{d}, J=7.3 \mathrm{~Hz}), 4.24-4.29(2 \mathrm{H}, \mathrm{m}), 3.65-3.76$ $(2 \mathrm{H}, \mathrm{m}), 3.38-3.41(2 \mathrm{H}, \mathrm{m}), 3.35-3.37(3 \mathrm{H}, \mathrm{m}), 3.22-3.29$ $(2 \mathrm{H}, \mathrm{m}) ;{ }^{13} \mathrm{C}$ NMR (150MHz, $\left.\mathrm{CD}_{3} \mathrm{OD}\right) \delta: 177.1$ (s), 165.0 (s), 160.8 (s), 158.4 (s), 156.6 (s), 154.6 (s), 132.6 (s), 130.6 (d), 120.6 (s), 115.1 (d), 104.4 (d), 102.5 (s), 99.6 (d), 97.6 (d), 93.7 (d), 81.5 (d), 76.9 (d), 76.2 (d), 76.1 (d), 73.2 (d), 70.0 (d), 69.4 (d), $65.3(\mathrm{t}), 60.3(\mathrm{t})$.

Kaempferol-3-O- $\beta$ - $D$-glucopyranosyl-7- $O-\alpha$ - L-rhamnopyranoside (7): $:^{[12]}$ white powder, $\mathrm{C}_{27} \mathrm{H}_{30} \mathrm{O}_{15},{ }^{1} \mathrm{H}$ NMR $(600 \mathrm{MHz}$, $\left.\mathrm{CD}_{3} \mathrm{OD}\right) \delta$ : $8.06(2 \mathrm{H}, \mathrm{d}, J=8.7 \mathrm{~Hz}), 6.89(2 \mathrm{H}, \mathrm{d}, J=8.7 \mathrm{~Hz})$ $6.68(1 \mathrm{H}, \mathrm{d}, J=2.0 \mathrm{~Hz}), 6.40(1 \mathrm{H}, \mathrm{d}, J=2.0 \mathrm{~Hz}), 5.55(1 \mathrm{H}, \mathrm{d}$, $J=1.4 \mathrm{~Hz}), 5.26(1 \mathrm{H}, \mathrm{d}, J=7.4 \mathrm{~Hz}), 3.38-4.49(10 \mathrm{H}, \mathrm{m}), 1.25$ $(3 \mathrm{H}, \mathrm{d}, J=6.5 \mathrm{~Hz}),{ }^{13} \mathrm{C}$ NMR $\left(150 \mathrm{MHz}, \mathrm{CD}_{3} \mathrm{OD}\right) \delta: 177.6$ (s), 161.5 (s), 160.7 (s), 160.1 (s), 156.9 (s), 155.9 (s), 133.6 (s), 131.0 (d), 120.9 (s), 114.3 (d), 105.6 (s), 100.6 (d), 100.2 (d), 99.3 (d), 94.6 (d), 77.5 (d), 76.6 (d), 74.1 (d), 71.6 (d), 70.2 (d), 70.1 (d), 70.0 (d), 69.6 (d), $60.8(\mathrm{t}), 18.1(\mathrm{q})$.

Herbacetin-8-O- $\beta$ - $D$-glucopyranoside (8): ${ }^{[12]}$ white powder, $\mathrm{C}_{21} \mathrm{H}_{20} \mathrm{O}_{12},{ }^{1} \mathrm{H}$ NMR $\left(600 \mathrm{MHz}, \mathrm{CD}_{3} \mathrm{OD}\right) \delta: 8.16(2 \mathrm{H}, \mathrm{d}, J=8.6$ $\mathrm{Hz}), 6.89(2 \mathrm{H}, \mathrm{d}, J=8.6 \mathrm{~Hz}), 6.60(1 \mathrm{H}, \mathrm{s}), 5.23(1 \mathrm{H}, \mathrm{d}, J=7.4$ $\mathrm{Hz}), 3.21-3.69(6 \mathrm{H}, \mathrm{m}) ;{ }^{13} \mathrm{C}$ NMR $\left(150 \mathrm{MHz}, \mathrm{CD}_{3} \mathrm{OD}\right) \delta: 177.4$ (s), 160.3 (s), 157.1 (s), 157.0 (s), 156.7 (s), 148.4 (s), 133.3 (s), 131.5 (d), 125.3 (s), 120.6 (s), 115.0 (d), 103.2 (s), 100.7 (d), 99.3 (d), 77.1 (d), 76.6 (d), 74.1 (d), 70.0 (d), 60.7 (t).

Rhodionin (9): ${ }^{[3]}$ greenish powder, $\mathrm{C}_{21} \mathrm{H}_{20} \mathrm{O}_{11},{ }^{1} \mathrm{H}$ NMR $(600$ $\left.\mathrm{MHz}, \mathrm{CD}_{3} \mathrm{OD}\right) \delta: 8.19(2 \mathrm{H}, \mathrm{d}, J=8.6 \mathrm{~Hz}), 6.89(2 \mathrm{H}, \mathrm{d}, J=8.6$ $\mathrm{Hz}), 6.62(1 \mathrm{H}, \mathrm{s}), 5.51(1 \mathrm{H}, \mathrm{d}, J=1.7 \mathrm{~Hz}), 4.13(1 \mathrm{H}, \mathrm{s})$, $3.42-3.84(3 \mathrm{H}, \mathrm{m}), 1.25(3 \mathrm{H}, \mathrm{d}, J=6.2 \mathrm{~Hz}) ;{ }^{13} \mathrm{C}$ NMR $(150$ 
Table 1 Agonistic activities of the isolated compounds on receptor $5-\mathrm{HT}_{2 \mathrm{~A}}{ }^{a}$

\begin{tabular}{ccc||ccc}
\hline Compounds & Concentration $/ \mathrm{mM}$ & Agonistic rate r $^{b} \%$ & Compounds & Concentration/mM & Agonistic rate $/ \%$ \\
\hline $\mathbf{1}$ & 0.97 & 9.2 & $\mathbf{9}$ & 1.01 & 17.3 \\
$\mathbf{2}$ & 0.95 & 22.5 & $\mathbf{1 0}$ & 0.96 & 7.3 \\
$\mathbf{3}$ & 1.01 & 16.7 & $\mathbf{1 1}$ & 0.98 & 4.6 \\
$\mathbf{4}$ & 1.03 & 18.2 & $\mathbf{1 2}$ & 1.02 & 33.5 \\
$\mathbf{5}$ & 0.97 & 22.4 & $\mathbf{1 3}$ & 1.03 & 42.1 \\
$\mathbf{6}$ & 1.01 & 8.0 & $\mathbf{1 4}$ & 0.96 & 46.2 \\
$\mathbf{7}$ & 0.99 & 18.4 & $\mathbf{1 5}$ & 0.98 & 37.6 \\
$\mathbf{8}$ & 1.03 & 23.1 & & & \\
\hline
\end{tabular}

${ }^{a}$ The positive control was serotonin hydrochloride $\left(\mathrm{EC}_{50} 8.53 \mathrm{nM}\right) .{ }^{b}$ The agonistic rate was percentage versus the positive control (normalized to $\left.100 \%\right)$ and was average of two independent tests.

$\left.\mathrm{MHz}, \mathrm{CD}_{3} \mathrm{OD}\right) \delta: 177.2(\mathrm{~s}), 160.5(\mathrm{~s}), 153.5$ (s), $151.5(\mathrm{~s}), 148.7$ (s), 145.8 (s), 137.1 (s), 130.9 (d), 128.4 (s), 123.6 (s), 116.2 (d), 106.1 (s), 100.6 (d), 99.0 (d), 73.4 (d), 72.1 (d), 71.5 (d), 71.0 (d), 18.0 (q).

Genistein (10): ${ }^{[13]}$ yellowish powder, $\mathrm{C}_{15} \mathrm{H}_{10} \mathrm{O}_{5},{ }^{1} \mathrm{H}$ NMR $(600$ $\left.\mathrm{MHz}, \mathrm{CD}_{3} \mathrm{OD}\right) \delta: 8.26(1 \mathrm{H}, \mathrm{s}), 7.27(2 \mathrm{H}, \mathrm{d}, J=8.5 \mathrm{~Hz}), 6.75(2 \mathrm{H}$, $\mathrm{d}, J=8.5 \mathrm{~Hz}), 6.39(1 \mathrm{H}, \mathrm{d}, J=2.0 \mathrm{~Hz}), 6.18(1 \mathrm{H}, \mathrm{d}, J=2.0 \mathrm{~Hz})$; ${ }^{13} \mathrm{C}$ NMR (150 MHz, $\left.\mathrm{CD}_{3} \mathrm{OD}\right) \delta: 180.5$ (s), 164.5 (s), 162.3 (s), 157.8 (s), 157.6 (s), 154.2 (d), 130.4 (d), 122.5 (s), 121.4(s), 115.4 (d), 104.6 (s), 99.2 (d), 93.9 (d)

Genistin (11): $:^{[14]}$ yellow powder, $\mathrm{C}_{21} \mathrm{H}_{20} \mathrm{O}_{10},{ }^{1} \mathrm{H}$ NMR $(600$ $\left.\mathrm{MHz}, \mathrm{DMSO}-\mathrm{d}_{6}\right) \delta: 8.31(1 \mathrm{H}, \mathrm{s}), 7.44(2 \mathrm{H}, \mathrm{d}, J=8.6 \mathrm{~Hz}), 7.05$ $(2 \mathrm{H}, \mathrm{d}, J=8.6 \mathrm{~Hz}), 6.33(1 \mathrm{H}, \mathrm{d}, J=2.1 \mathrm{~Hz}), 6.17(1 \mathrm{H}, \mathrm{d}, J=2.1$ $\mathrm{Hz}), 4.87(1 \mathrm{H}, \mathrm{d}, J=7.1 \mathrm{~Hz}), 3.10-3.70(6 \mathrm{H}, \mathrm{m}) ;{ }^{13} \mathrm{C}$ NMR $(150$ $\mathrm{MHz}, \mathrm{DMSO}_{\mathrm{d}}$ ) $\delta: 154.7$ (d), 122.5 (s), 180.5 (s), 162.3 (s), 99.4 (d), 163.9 (s), 94.2 (d), 157.7 (s), 105.4 (s), 124.3 (s), 130.2 (d), 116.0 (d), 157.3 (s), 100.2 (d), 73.3 (d), 76.7 (d), 69.7 (d), $77.2(\mathrm{~d}), 60.7(\mathrm{t})$.

Tyrosol (12): ${ }^{[15]}$ white powder, $\mathrm{C}_{8} \mathrm{H}_{10} \mathrm{O}_{2},{ }^{1} \mathrm{H}$ NMR $\left(600 \mathrm{MH}_{\mathrm{Z}}\right.$, $\left.\mathrm{CD}_{3} \mathrm{OD}\right) \delta: 7.07(2 \mathrm{H}, \mathrm{d}, J=8.3 \mathrm{~Hz}), 6.66(2 \mathrm{H}, \mathrm{d}, J=8.3 \mathrm{~Hz})$, $3.75(2 \mathrm{H}, \mathrm{t}, J=7.1 \mathrm{~Hz}), 2.78(2 \mathrm{H}, \mathrm{t}, J=7.1 \mathrm{~Hz}) ;{ }^{13} \mathrm{C}$ NMR $(150$ $\left.\mathrm{MH}_{\mathrm{Z}}, \mathrm{CD}_{3} \mathrm{OD}\right) \delta: 156.5$ (s), 130.8 (s), 130.6 (d), 116.1 (d), 64.5 $(\mathrm{t}), 39.2(\mathrm{t})$.

Salidroside (13): ${ }^{[16]}$ white powder, $\mathrm{C}_{14} \mathrm{H}_{20} \mathrm{O}_{7},{ }^{1} \mathrm{H}$ NMR $(600$ $\left.\mathrm{MH}_{\mathrm{z}}, \mathrm{CD}_{3} \mathrm{OD}\right) \delta: 7.07(2 \mathrm{H}, \mathrm{d}, J=8.3 \mathrm{~Hz}), 6.68(2 \mathrm{H}, \mathrm{d}, J=8.3$ $\mathrm{Hz}), 4.31(1 \mathrm{H}, \mathrm{d}, J=7.8 \mathrm{~Hz}), 4.01-4.04(1 \mathrm{H}, \mathrm{m}), 2.84-3.05$ $(3 \mathrm{H}, \mathrm{m}) ;{ }^{13} \mathrm{C}$ NMR $\left(150 \mathrm{MH}_{\mathrm{z}}, \mathrm{CD}_{3} \mathrm{OD}\right) \delta: 156.9$ (s), 130.9 (d), 130.8 (s), 116.2 (d), 104.5 (d), 78.2 (d), 78.0 (d), 75.2 (d), 72.2 $(\mathrm{t}), 71.7(\mathrm{~d}), 62.8(\mathrm{t}), 36.5(\mathrm{t})$.

Rosin (14): ${ }^{[12]}$ white powder, $\mathrm{C}_{15} \mathrm{H}_{20} \mathrm{O}_{6},{ }^{1} \mathrm{H}$ NMR $(600 \mathrm{MHz}$, $\left.\mathrm{CD}_{3} \mathrm{OD}\right) \delta: 7.39-7.41(1 \mathrm{H}, \mathrm{m}), 7.26-7.27(2 \mathrm{H}, \mathrm{m}), 7.21-7.24$ $(2 \mathrm{H}, \mathrm{m}), 6.65(1 \mathrm{H}, \mathrm{d}, J=16.1 \mathrm{~Hz}), 6.30-6.34(1 \mathrm{H}, \mathrm{m}), 4.51(1 \mathrm{H}$, $\mathrm{d}, \mathrm{J}=7.5 \mathrm{~Hz}), 4.29-4.36(2 \mathrm{H}, \mathrm{m}), 3.67-3.87(2 \mathrm{H}, \mathrm{m})$, 3.32-3.38 (4H, m); ${ }^{13} \mathrm{C}$ NMR (150 MHz, CD $\left.\mathrm{CD}_{3} \mathrm{OD}\right) \delta: 137.3$ (s), 132.7 (d), 128.7 (d), 127.6 (d), 126.5 (d), 125.8 (d), 102.5 (d), 77.1 (d), 77.0 (d), 74.2 (d), 70.8 (d), 69.9 (t), 61.6 (t).

Rosavin (15): ${ }^{[12]}$ white powder, $\mathrm{C}_{20} \mathrm{H}_{28} \mathrm{O}_{10},{ }^{1} \mathrm{H}$ NMR $(600$ $\left.\mathrm{MHz}, \mathrm{CD}_{3} \mathrm{OD}\right) \delta: 7.40-7.44(2 \mathrm{H}, \mathrm{m}), 7.28-7.29(2 \mathrm{H}, \mathrm{m})$, $7.19-7.23(1 \mathrm{H}, \mathrm{m}), 6.68(1 \mathrm{H}, J=16.3 \mathrm{~Hz}), 6.30-6.35(1 \mathrm{H}, \mathrm{m})$, 4.50-4.51 $(1 \mathrm{H}, \mathrm{m}), 4.29-4.32(1 \mathrm{H}, \mathrm{m}), 4.37(1 \mathrm{H}, \mathrm{d}, J=7.6$ $\mathrm{Hz}), 4.34(1 \mathrm{H}, \mathrm{d}, J=7.2 \mathrm{~Hz}), 4.07-4.11(1 \mathrm{H}, \mathrm{m}), 3.74-3.85$ $(3 \mathrm{H}, \mathrm{m}), 3.24-3.57(6 \mathrm{H}, \mathrm{m}) ;{ }^{13} \mathrm{C}$ NMR $\left(150 \mathrm{MHz}, \mathrm{CD}_{3} \mathrm{OD}\right) \delta$ : 138.5 (s), 133.8 (d), 129.7 (d), 128.7 (d), 127.6 (d), 126.8 (d), 103.5 (d), 105.2 (d), 78.1 (d), 77.0 (d), 75.1 (d), 74.3 (d), 72.5 (d), $71.8(\mathrm{~d}), 70.9(\mathrm{t}), 69.6(\mathrm{~d}), 69.5(\mathrm{t}), 66.8(\mathrm{t})$.

Pharmacological studies demonstrated that the selective agonists of serotonin $(5-\mathrm{HT})$ receptor $5-\mathrm{HT}_{2 \mathrm{~A}}$ were closely involved in the therapy of hypertension, hyperlipidemia and obesity. ${ }^{[5,6]}$ Consequently, these isolates from the title plant were further assayed for their agitating activities on $5-\mathrm{HT}_{2 \mathrm{~A}}$ receptor on HEK293 cell lines in vitro.

As shown in Table 1, the bioassay results revealed that all the test compounds exhibit varying agitating activities against the $5-\mathrm{HT}_{2 \mathrm{~A}}$ receptor. Among them, rosin (14) exhibits the strongest effect with an agonistic rate of $46.2 \%$ at the concentration of $0.96 \mathrm{mM}$, following by salidroside (13), rosavin (15) and tyrosol (12) with agonistic rates of $42.1 \%, 37.6 \%$ and $33.5 \%$ at about $1.00 \mathrm{mM}$, respectively.

In conclusion, 11 flavonoids, 2 phenylethanoids and 2 phenylpropanoids were isolated from the roots of $R$. rosea $\mathrm{L}$. Among them, flavones $\mathbf{1}$ and $\mathbf{2}$ were isolated from the Rhodiola genus for the first time. The bioassay results revealed that rosin (14), salidroside (13), rosavin (15) and tyrosol (12) are probably the main lipid-lowering components of $R$. rosea L., which may provide additional information for the further development of this medicine-food resource plant.

\section{Acknowledgments}

We thank the Natural Science Foundation of the Jiangsu Higher Education Institutions of China (No. 18KJB350011) and the Natural Research Foundation for Advanced Talents of Taizhou university (Nos. QD2016010 and QD2016044) for the financial support.

\section{Supporting Information}

The bioassay results of extracts and fractions on receptor 5-HT2A are available in Supporting Information.

\section{Conflict of Interest}

The authors declare no conflict of interest.

Copyright (c) 2020 Hao Wang, Ji-Lei Liang, Qing-Ming He, Guo-Hai Zhou, Cun-Fu Li, Ping-He Wei, and Hao-Yan Dong. This article is an open access article distributed under the terms and conditions of the Creative Commons Attribution (CC BY) license (http://creativecommons.org/licenses/by/4.0/). The use, distribution or reproduction in other forums is permitted, provided the original author(s) or licensor are credited and that the original publication in this journal is cited, in accordance with accepted academic practice. No use, distribution or reproduction is permitted which does not comply with these terms.

\section{References}

[1] Sun, J.; Zhou, W. H.; Gu, T. W.; Zhu, D. L.; Bi, Y. A retrospective study on association between obesity and cardiovascular risk diseases with aging in Chinese adults. Sci. Rep. 2018, 8 5806-5813.

[2] Cheng, T. T.; Yuan, X. B.; Hui, X. H.; Ke, W. D.; Guo, H. B. Research progress on chemical constituents and lipid-lowering mechanism of alkaloids in Nelumbinis folium. Chin. Tradit. Herb. Drugs 2019, 50, 1998-2003.

[3] Diermen, D. V.; Marston A.; Bravo, J.; Reist, M.; Carrupt, P. A.; Hostettmann, K. Monoamine oxidase inhibition by Rhodiola rosea L. roots. J. Ethnopharmacol. 2009, 122, 397-401.

[4] Kwon, H. J.; Ryu, Y. B.; Jeong, H. J.; Kim, J. H.; Park, S. J.; Chang, J. S.; Kim, M.; Rho, M. C.; Lee, W. S. Rhodiosin, an antioxidant flavonol glycoside from Rhodiola rosea. J. Korean Soc. Appl. Biol. 
Chem. 2009, 52, 486-492.

[5] Munusamy, V.; Yap, B. K.; Buckle, M. J. C.; Doughty, S. W.; Chung, L. Y. Structure-based identification of aporphines with selective 5- $\mathrm{HT}_{2 \mathrm{~A}}$ receptor-binding activity. Chem. Biol. Drug Des. 2013, 81, 250-256.

[6] Hurt, R. T.; Edakkanambeth, V. J.; Ebbert, J. O. New pharmacological treatments for the management of obesity. Curr. Gastroenterol. Rep. 2014, 16, 1-8.

[7] Wang, H.; Geng, C. A.; Xu, H. B.; Huang, X. Y.; Ma, Y. B.; Yang, C. Y.; Zhang, X. M.; Chen, J. J. Lignans from the fruits of Melia toosendan and their agonistic activities on melatonin receptor $\mathrm{MT}_{1}$. Planta Med. 2015, 81, 847-854.

[8] Wang, L.; Li, H. Y.; Liang, N.; Chen, N. H. Isolation and identification of the flavonoids constituents from Dryopteris championii. Nat. Prod. Res. Dev. 2020, 32, 778-782.

[9] Han, J. T.; Bang, M. H.; Chun, O. K.; Kim, D. O.; Lee, C. Y.; Baek, N. I. Flavonol glycosides from the aerial parts of Aceriphyllum rossil and their antioxidant activities. Arch. Pharm. Res. 2004, 27, 390-395.

[10] Nakatani, M.; Shimokoro, M.; Zhou, J. B.; Okamura, H.; Iwagawa, T.; Tadera, K.; Nakayama, N.; Min, H.; Naoki, G. Limonoids from Melia toosendan. Phytochemistry 1999, 52, 709-714.

[11] Ma, C. Y.; Hu, L. M.; Fu, Q. Y.; Gu, X. H.; Tao, G. J.; Wang, H. X. Separation of four flavonoids from Rhodiola rosea by on-line combination of sample preparation and counter-current chromatography. J. Chromatogr. A 2013, 1306, 12-19.

[12] Elizabeth, M.; Daise, L. L.; Paula, N. B.; Andreas, S. Purification of phenylalkanoids and monoterpene glycosides from Rhodiola rosea L. roots by high-speed counter-current chromatography. Phytochem. Anal. 2013, 24, 129-134.

[13] Murthy, M. S. R.; Rao, E. V. Carbon-13 nuclear magnetic resonance spectra of isoflavones. Magn. Res. Chem. 1986, 24, 225-230.

[14] Nishanbaev, S. Z.; Bobakulov, K. M.; Narbutaeva, D. A.; Aripova, S. F.; Khushbaktova, Z. A.; Abdullaev, N. D. Flavonoids from the aerial part of Alhagi persarum of the flora of uzbekistan and their biological activity. Chem. Nat. Compd. 2020, 56, 729-731.

[15] Xu, Y. L.; Zhu, W. Y.; Tan, J. L.; Zhang, H. Chemical constituents of Leontopodium haplophylloides and their anti-bacterial activities. Nat. Prod. Res. Dev. 2019, 31, 441-445.

[16] Gao, B. B.; She, G. M.; She, D. M. Chemical constituents and biological activities of plants from the genus Ligustrum. Chem. Biodivers. 2013, 10, 96-128. 